\title{
A molecular dynamics study of complexes of poly(glutamate) and dodecyltrimethylammonium
}

\author{
David Zanuy ${ }^{*}$ and Carlos Alemán \\ Departament d'Enginyeria Química, E.T.S. d'Enginyers Industrials de Barcelona, \\ Universitat Politècnica de Catalunya, Diagonal 647, \\ Barcelona E-08028, Spain
}

Corresponding authors: david.zanuy@upc.edu and carlos.aleman@upc.edu

\begin{tabular}{ccc}
\hline$\#$ & Page & Description \\
\hline Figure S1 & S2 & For all the simulation series evolution of the Root Mean Square Deviation \\
Figure S2 & S3 & Spatio-temporal evolution of the hydrogen bonds in the $17 / 5$ helix \\
Figure S3 & S4 & Snapshots corresponding to the simulations of the $17 / 5$ helix \\
Figure S4 & S5 & Snapshots corresponding to the simulations of the $5 / 2$ helix \\
Figure S5 & S6 & Snapshots corresponding to the simulations of the $2 / 1$ helix \\
Figure S6 & S7 & Conformational distribution of the surfactant hydrophobic tails
\end{tabular}


(a)

$\mathrm{FF}_{1}$ - cut off 14

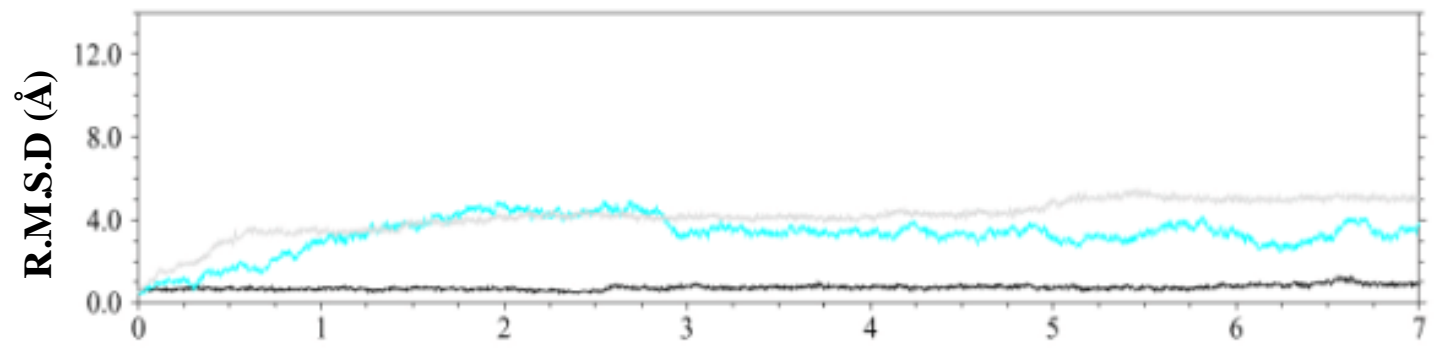

$\mathrm{FF}_{2^{-}}$cut off 14

(b)

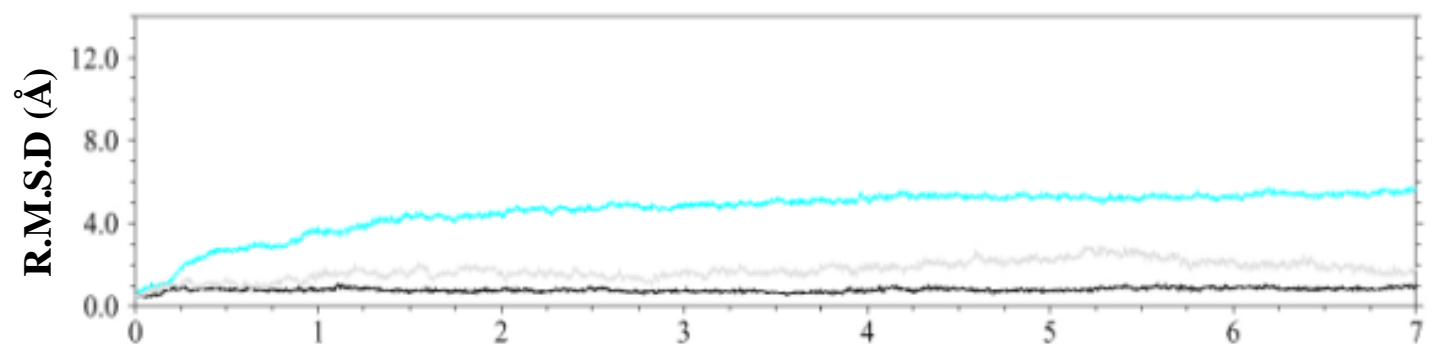

(c)

$\mathrm{FF}_{1}$ - PME

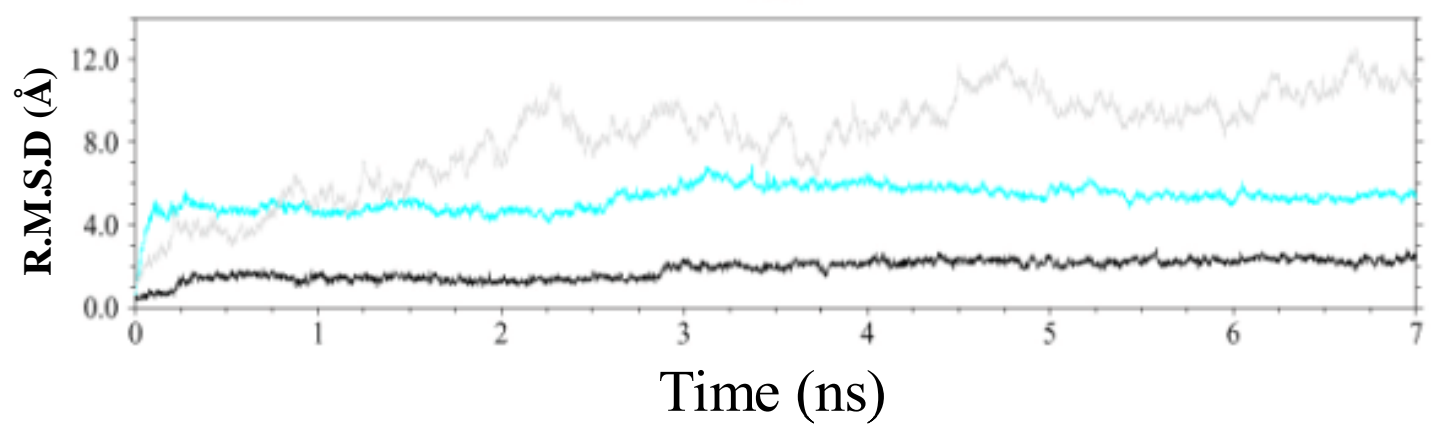

Figure S1. For all the simulation series evolution of the Root Mean Square Deviation (RMSD; in $\AA$ ). Results correspond to the different approaches used to estimate the electrostatic potential: (a) spherical cut off $14 \AA$ and FF1 (series I); (b) spherical cut off $14 \AA ̊$ and FF2 (series II) and (c) PME and FF1 (series III). The starting conformations of the polypeptide chain in the simulations were the 17/5 (black), 5/2 (grey) and 2/1 (light blue) helices. 
(a)

(b)

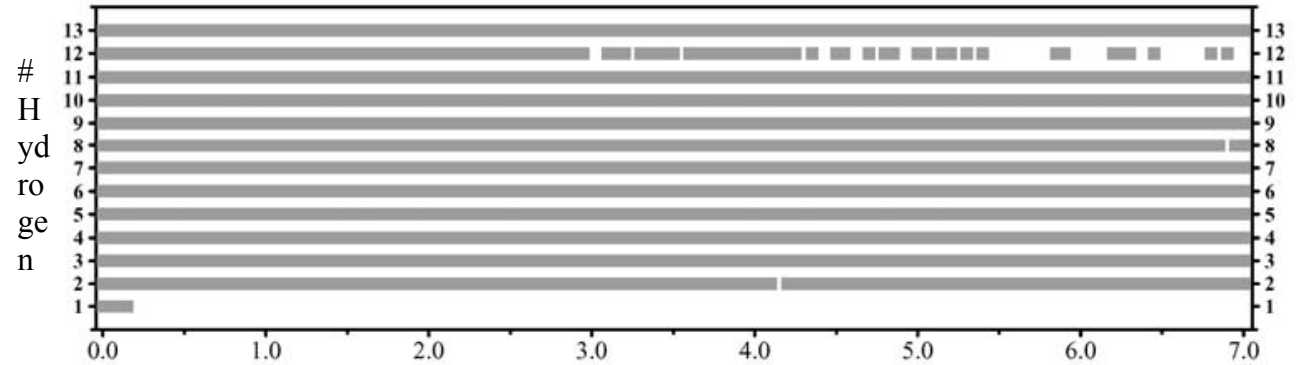

(c)
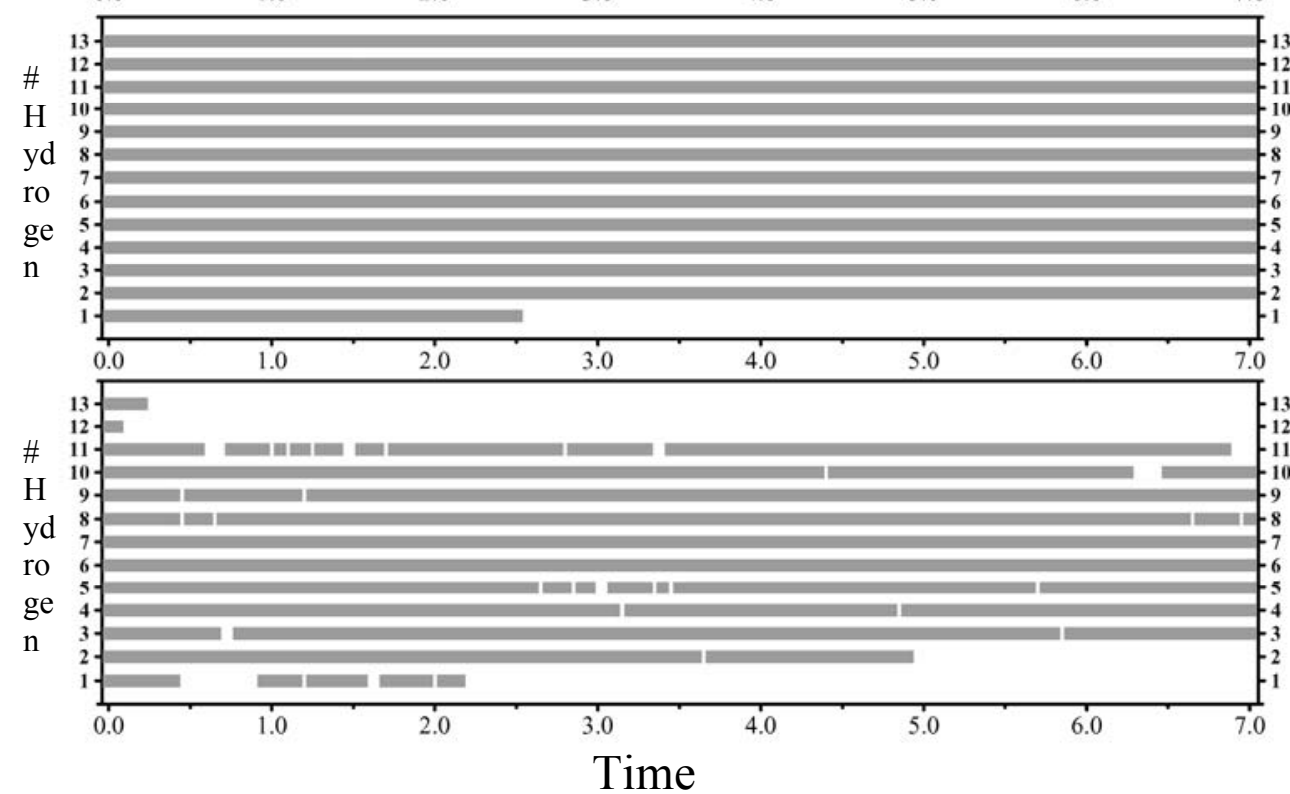

Figure S2. For the simulations starting from the $17 / 5$ helix, spatio-temporal evolution of the $\mathrm{N}-\mathrm{H}(\mathrm{i}) \rightarrow \mathrm{O}=\mathrm{C}(i+3)$ type hydrogen bonds in the PGGA chain. Results correspond to the different approaches used to estimate the electrostatic potential: (a) spherical cutoff $14 \AA$ and FF1 (simulation Ib); (b) spherical cutoff $14 \AA$ and FF2 (simulation IIb) and (c) PME and FF1 (simulation IIIb). 


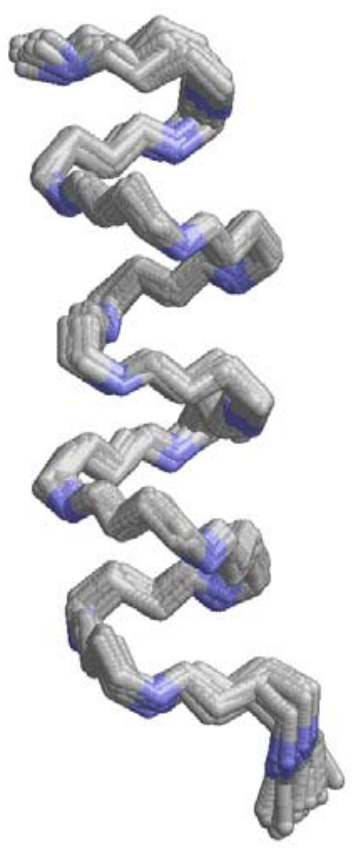

(a)

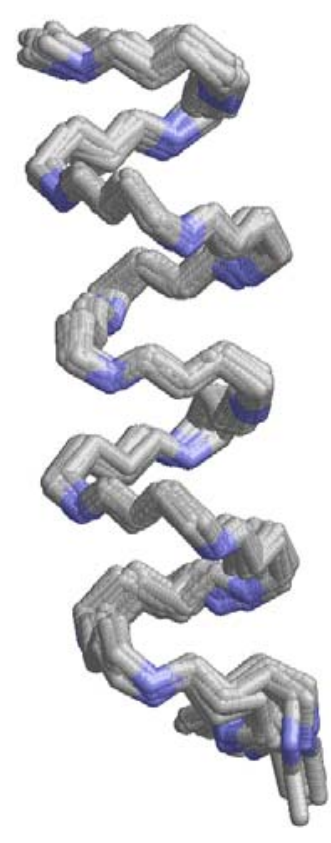

(b)

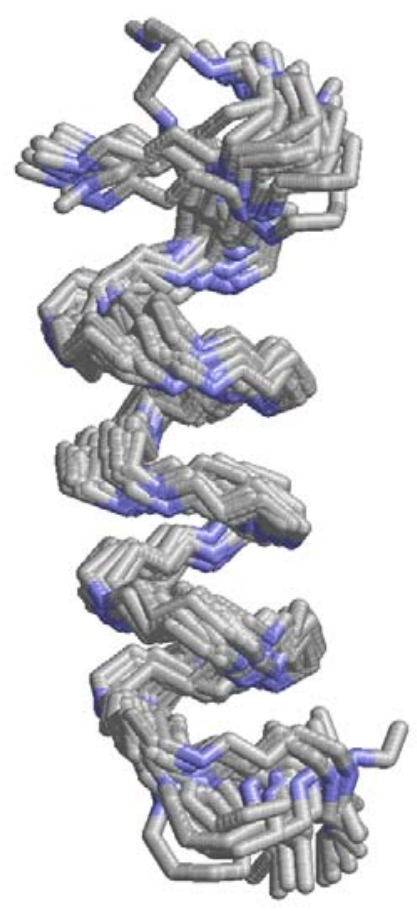

(c)

Figure S3. Superposition of equality spaced snapshots corresponding to the simulations starting from the $\alpha$-like helix (17/5). From left to right the results obtained in the simulation Ia (a), IIa (b) and IIIa (c), respectively. 


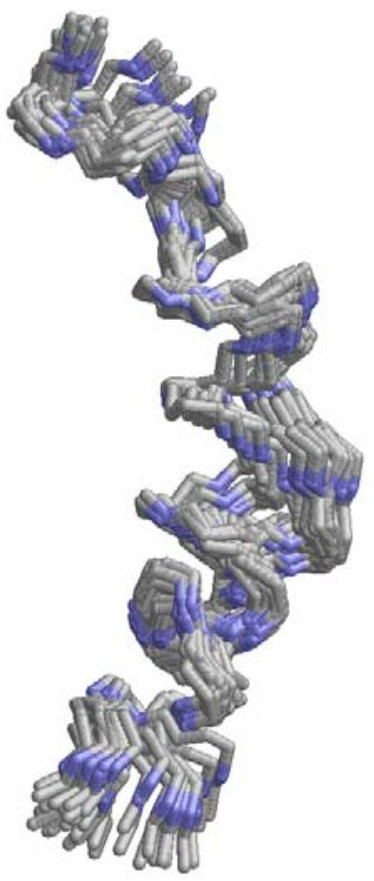

(a)

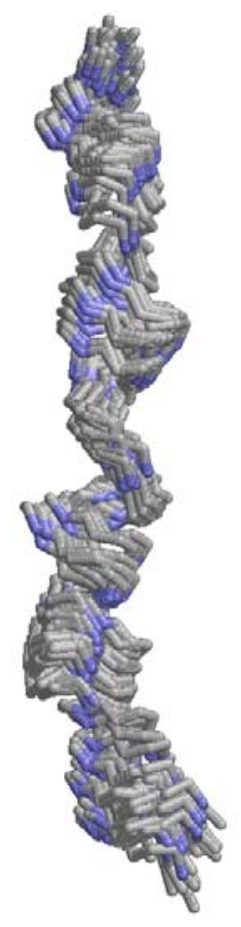

(b)

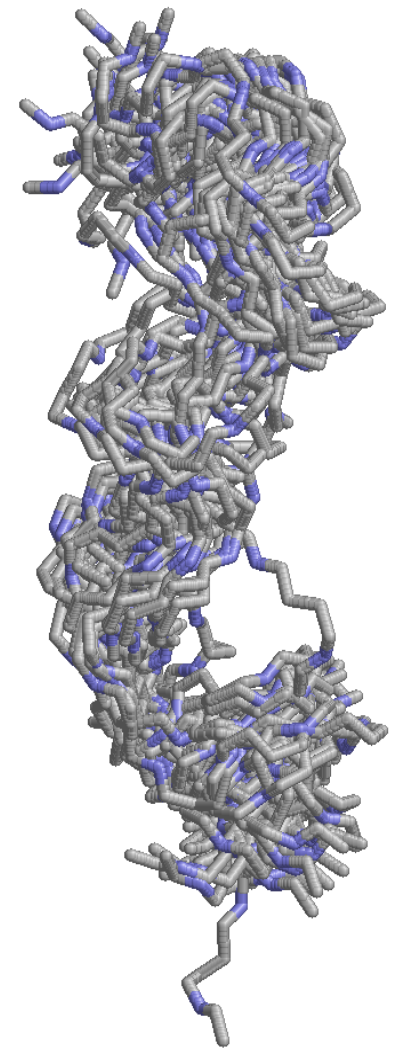

(c)

Figure S4. Superposition of equality spaced snapshots corresponding to the simulations starting from the helix $5 / 2$. From left to right the results obtained in the simulations $\mathrm{Ib}$ (a), IIb (b) and IIIb (c), respectively. 


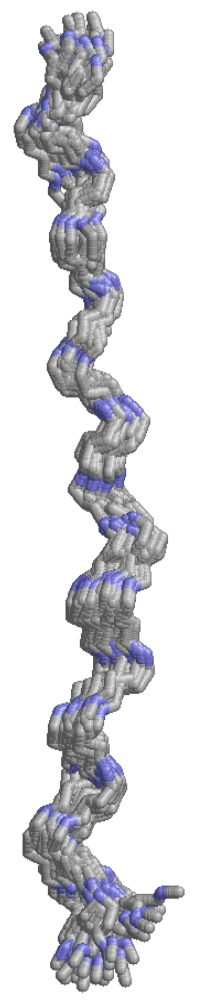

(a)

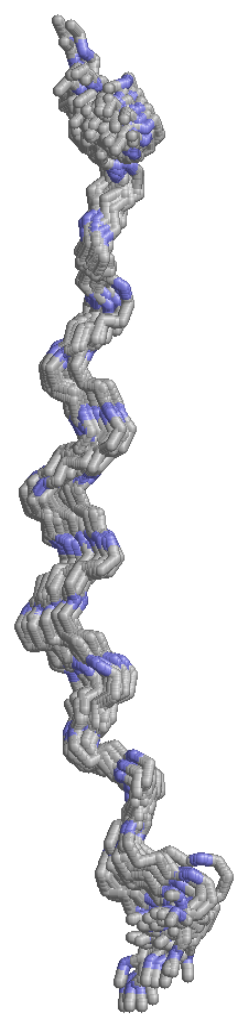

(b)

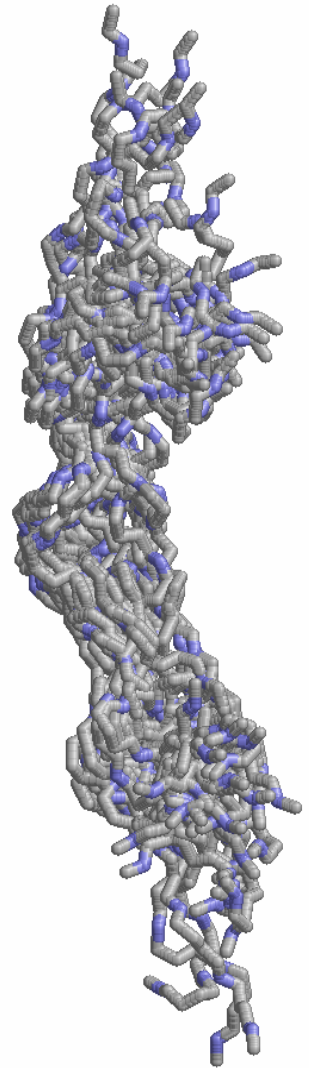

(c)

Figure S5. Superposition of equality spaced snapshots corresponding to the simulations starting from the helix $2 / 1$. From left to right the results obtained in the simulations Ic (a), Iic (b) and IIIc (c), respectively. 

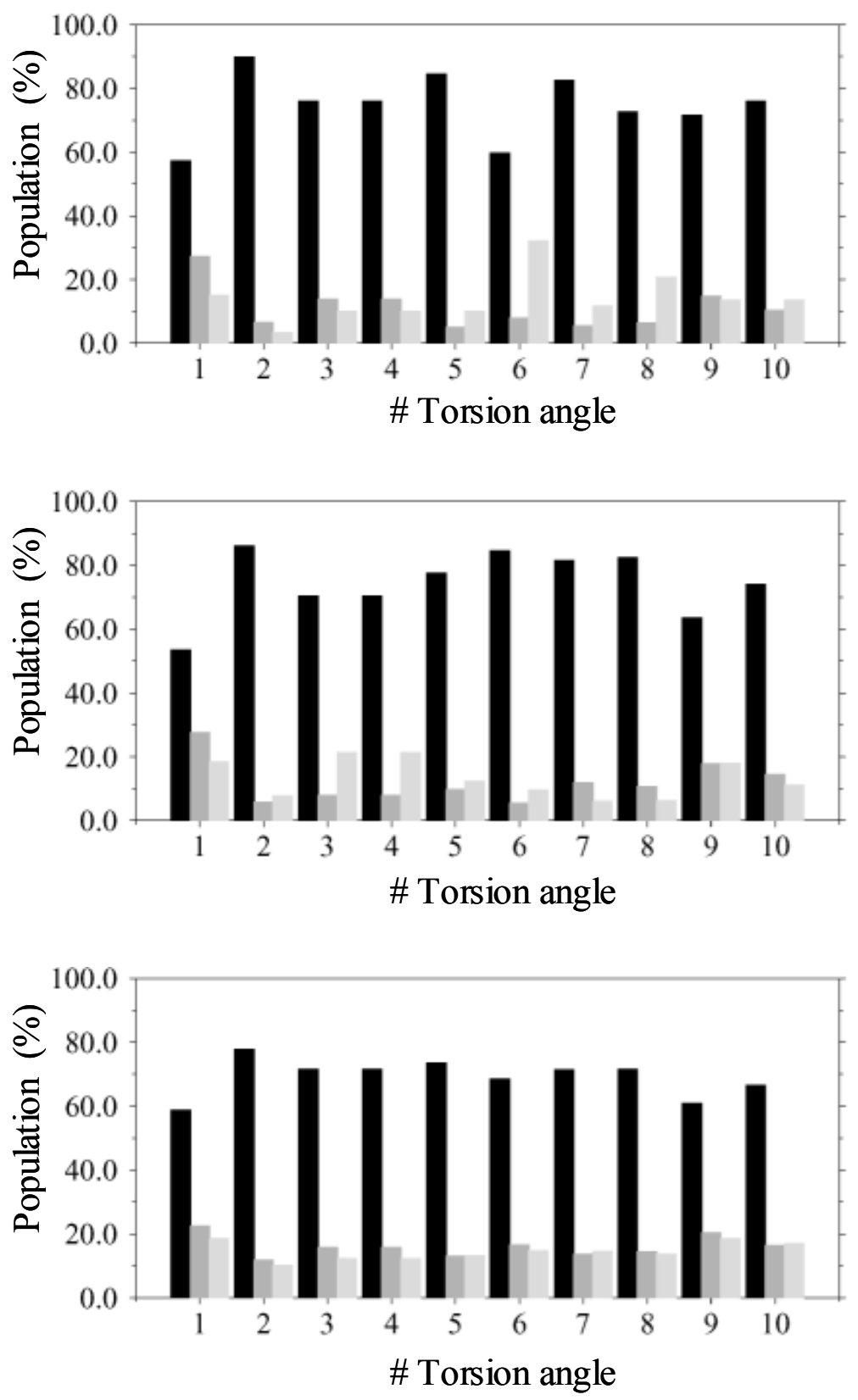

Figure S6. Conformational distribution of the surfactant hydrophobic tails depending on the dihedral angle position, starting from the positively charged head. The percentage of trans (black), gauche (dark gray) and gauche (light gray) rotamers are depicted for the $17 / 5$ helical conformation of the polypeptide chain and for each simulation series (see Table 1): (a) simulation Ia; (b) simulation IIa; and (c) simulation IIIa. 